\title{
La vulnerabilidad como economización de lo social en el campo de políticas sociales vinculadas a familias
}

\author{
Mónica De Martino*
}

\section{RESUMEN}

El presente artículo intenta problematizar una de las categorías rectoras presentes, desde hace unas dos décadas, en el diseño e implementación de Políticas Sociales referidas a infancia y familia. Lo hace retomando la forma más elaborada de su definición en nuestro país. Para ello puntualizaremos algunas escuelas de pensamiento que se encuentran subyacentes en tal forma de concebir, de cierta manera a partir de una razón instrumental, la vulnerabilidad social.

Palabras clave: Vulnerabilidad - infancia - familia - políticas sociales.

\section{A vulnerabilidad como economización do social no campo de políticas sociais vinculadas a famílias}

\begin{abstract}
RESUMO
O presente artigo tenta problematizar uma das categorias rectoras presentes, desde faz umas duas décadas, no desenho e implementación de Políticas Sociais referidas a infância e família. Fá-lo retomando a forma mais elaborada de sua definição em nosso país. Para isso puntualizaremos algumas escolas de pensamento que se encontram subyacentes em tal forma de conceber, de certa maneira a partir de uma razão instrumental, a vulnerabilidad social
\end{abstract}

Palavras chave: Vulnerabilidad - infância - família - políticas sociais

* Uruguaya. Trabajadora Social. Docente Departamento de Trabajo Social. Universidad de la República. Coordinadora del Programa de Doctorado en Trabajo Social. Coordinadora del Área de Infancia y Familia. Correo electrónico: monicad@fcs.edu.uy 


\section{The vulnerability and economization of the social in the field of social policies related to families}

\section{ABSTRACT}

This article attempts to problematize the categories of lead present, for about two decades in the design and implementation of social policies regarding children and family. What does taking the more elaborate form of its definition in our country. It pointed to some schools of thought that are behind this way of conceiving of a way from an instrumental reason, the social vulnerability.

Key words: Vulnerability - child - family - social policies. 


\section{Antecedentes}

Hace ya cierto tiempo que manifestamos preocupación académica por una de las formas más refinadas de definir la vulnerabilidad en nuestro país. Especialmente cuando tales elaboraciones impregnan políticas y programas sociales. Y tal preocupación aumenta en la medida que percibimos una suerte de negligencia, por parte de nuestra disciplina, con relación a la necesaria búsqueda de los orígenes de la misma. Estas ausencias nos permiten indicar que existe, con excepciones, una suerte de apropiación indebida por parte del colectivo profesional, en la medida que éste manifiesta cierta negligencia en la búsqueda de la génesis de tal definición y las escuelas de pensamiento que subyacen a ella.

\section{El concepto de vulnerabilidad en cuestión}

Es habitual en las ciencias humanas el auge y posterior devaluación de algunos conceptos o categorías teóricas. Muchas veces apelamos a los conceptos de vulnerabilidad -exclusión- integración para oponernos a los indicadores utilizados comúnmente para medir la pobreza. ${ }^{1}$

Pero pobreza y exclusión-vulnerabilidad refieren a distintos procesos societales. Mientras la pobreza tiene que ver con la desigualdad en la apropiación de la riqueza, la exclusión y la vulnerabilidad se definen en función de la debilidad de los lazos de integración social (Castel, 1997).

Desigualdad e inequidad hablan de dimensiones políticas y económicas, mientras que exclusión-vulnerabilidad, si bien relacionadas con procesos macroscópicos propios del capitalismo tardío, hacen referencia a procesos de orden cultural y social, remiten a aquel constante objeto de preocupación de la sociología francesa: la cohesión social y su potencial opuesto, la anomia o desintegración social. "El concepto de exclusión, así como el de vulnerabilidad, anuncia y denuncia una realidad distinta. Estaría dando cuenta de nuevos problemas en la integración social, estaría planteando una nueva versión de la integración social" (Baraibar, 1999).

1 Para una crítica de las modalidades de medición de la pobreza: De los Campos, H. El Índice de Necesidades básicas insatisfechas. Crítica de la definición oficial y propuesta de una metodología alterna. Documento de Trabajo Nro. 13. FCS - DTS - UDELAR. Montevideo. 2000. 
En nuestro país, en el campo de las Ciencias Sociales colocaremos énfasis en la manera de entender la vulnerabilidad, que se presenta como la forma tal vez refinada y rigurosa, pero que a nuestro entender presenta ciertas debilidades y abreva en escuelas de pensamiento que, consideramos, abordan a individuos y familias de una manera un tanto abstracta y formal.

En la literatura vernácula, el equipo liderado por Rubén Kaztman, desde una perspectiva que se acerca al asset vulnerability framework norteamericano, intenta analizar los procesos de pobreza, exclusión y vulnerabilidad social desde una perspectiva particular.

La intencionalidad de estos autores -y de otros- consiste en "captar mejor la dinámica de reproducción de los sistemas de desigualdad social, de las condiciones de marginalidad y exclusión, al mismo tiempo que ofrece un instrumental analítico más potente para la acción" (Kaztman, Beccaria, Filgueira, Kessler y Golbert, 1999:2).

Esta propuesta analítica intenta colocar en un lugar más activo a los beneficiarios de las Políticas Sociales, tratando de identificar sus recursos, ya sean éstos utilizados o utilizables para proveer bienestar social.

Teóricamente, intenta identificar el "portafolio de activos" de individuos y hogares con el objetivo de reconocer sus estrategias de vida, la forma en que administran los riesgos de diversa índole ${ }^{2}$ y los mecanismos de promover su propio bienestar. Definiendo tales activos de la siguiente manera: "Los activos sociales de un hogar se componen del conjunto de recursos que en una instancia dada pueden ser movilizados en busca de mejoras en el bienestar o de evitar caídas en el nivel de bienestar de un hogar" (Katzman, Beccaría, Filgueira, Kessler E Golbert, 1999: 9).

Del mismo modo, intenta identificar los "pasivos", de individuos y hogares, es decir, los obstáculos o debilidades que éstos poseen para colocar en acción tales activos.

Identifican los autores citados ut supra tres tipos de activos básicos: el capital físico, el capital humano y el capital social. El primero de ellos hace referencia tanto al capital financiero como al capital propiamente físico. En tanto el capital humano nos remite al trabajo,

2 Sobre el concepto de Riesgo remitimos: Beck, U. (1988). 
la salud y la educación. Por último, el capital social nos habla de las redes de reciprocidad en las cuales se insertan individuos y hogares, los lazos afectivos, habilidades y destrezas sociales y el acceso a la información.

Pero estos activos no son analizados como elementos inmóviles, sino en el juego de relaciones que mantienen con la "estructura de oportunidades" que la sociedad otorga a tales individuos y familias, estructura que por definición es variable de acuerdo a las circunstancias socio-históricas. "Las estructuras de oportunidades se definen como probabilidades de acceso a bienes, a servicios o al desempeño de actividades. Estas oportunidades inciden sobre el bienestar de los hogares, ya sea porque permiten o facilitan a los miembros del hogar el uso de sus propios recursos o porque los proveen otros nuevos" (Kaztman, 1999:21).

Tal estructura de oportunidades obviamente no es una entelequia, una mera abstracción, por el contrario, es la conjugación del mercado, el Estado y la sociedad. Los autores dirán: “De la comparación entre los activos y los requerimientos de acceso a las estructuras de oportunidades resulta una evaluación del nivel de vulnerabilidad de las personas, que varía inversamente a su capacidad para superar las barreras al logro de su bienestar" (Katzman, Beccaría, Filgueira, Kessler y Golbert, 1999: 93).

Este enfoque de la vulnerabilidad identifica además cambios morfológicos en el fenómeno de la pobreza. Tales cambios en la morfología de la pobreza se relacionarían, según los autores referenciados, con el bajo capital humano de individuos y familias que no aseguran una inserción estable en el mercado de trabajo. En proyección, auguran la debilidad de los lazos familiares y la reproducción intergeneracional de la pobreza.

Obviamente, más allá de posibles activos y su identificación e incentivos por parte de diversos programas sociales e intervenciones técnicas, cabe señalar que la estructura de oportunidades en nuestro país también ha sufrido cierto adelgazamiento, acorde con el "adelgazamiento" del Estado indicado por O’Malley (1991), autor vinculado a la corriente anglosajona denominada governmentality studies. Cabe resaltar que tal corriente de pensamiento intenta reunir los enfoques marxistas y foucaultianos para analizar las formas de ejercicio del poder estatal en las sociedades del capitalismo tardío, 
diferenciándose de manera obvia con los autores nacionales hasta ahora analizados.

Ese adelgazamiento de la estructura de oportunidades se explica, entre diversas razones porque: "Los niveles educativos para alcanzar ingresos razonables se han incrementado, el acceso a la salud de calidad se ha estratificado, el logro de empleos no precarios se ha dificultado y los lazos familiares se han debilitado. Estado, mercado y sociedad conspiran para la delimitación de un amplio sector de población con un portafolio de activos precario, incompleto y en última instancia frágil para hacer frente a las situaciones adversas o ajustarse al ciclo vital y las vulnerabilidades asociadas a sus diferentes etapas" (Katzman, Beccaría, Filgueira, Kessler y Golbert, 1999, p. 55).

Desde esta perspectiva, los autores pretenden afinar el concepto de vulnerabilidad social, en cierta medida difuso y banalizado, entendiéndolo como una suerte de relación entre los activos disponibles, ya sean éstos individuales o familiares, y la cartera de oportunidades que otorgan mercado, Estado y sociedad. Y lo hacen a través de refinadas relaciones entre variables, indicadores e índices.

Desde nuestra perspectiva, la propuesta de los autores, a quienes reconocemos su compromiso y densidad intelectual, aborda fenómenos complejos a partir de una perspectiva altamente formal y abstracta. En otras palabras, introducen una suerte de economización de lo social. Intentaremos fundamentar nuestra afirmación.

\section{Limitaciones que surgen de la génesis de tal perspectiva}

Como ya es sabido, el proyecto sociológico de Durkheim, plasmado a lo largo de sus obras, era distinguir claramente esa nueva disciplina llamada Sociología de la Psicología y de la Economía, tanto en términos de su objeto como de su arsenal teórico-metodológico. Al contrario de análisis individualistas, la sociedad era considerada por Durkheim como una entidad sui generis y no apenas un mero agrado de sus componentes. Este abordaje ve el raciocinio sociológico como algo simplemente extraño al cuadro analítico de individuos que racionalmente persiguen sus intereses. Si la sociología ha sido 
influenciada por Durkheim desde hace ya mucho tiempo, es cierto que existe una suerte de consenso entre diversos teóricos. Basta pensar en su totalidad las trayectorias y obras de Parsons, Bourdieu y Giddens, por ejemplo, que indican la irreductibilidad de la vida social a la lógica económica.

Pero el surgimiento de la teoría del Elección Racional, en la década de los ochenta, basada en obras de diversos autores (Becker, 1976; Coleman, 1990; Olson, 1965), es en cierto modo sorprendente, en la medida que puede resumirse como la subordinación del homo sociologicus al homo economicus, y algo de ello está presente incluso en el lenguaje utilizado por Katzman et al. (1999).

Esta teoría aparece como una expresión refinada para capturar los diversos aspectos de la vida social, desde la frecuencia de la pertenencia a cultos religiosos, hasta fenómenos más vinculados a la vida privada: padrones de casamientos, suicidios, etc. Y, es más, esta teoría puede definirse como una teoría que propone explicar el comportamiento social y político partiendo de que las personas actúan siempre racionalmente.

Pero detengámonos en los problemas de la teoría de la escuela racional:

- La distinción entre actuar como si fuese racional y actuar racionalmente: Los teóricos de la elección racional defienden sus teorías argumentando que la idea de que las personas actúan racionalmente es confirmada y comprobada empíricamente. Pero: ¿qué tienen en mente cuando hablan de corroboración o confirmación empírica? Los autores refieren al hecho de que el modelo que resulta de sus perspectivas permite previsiones precisas sobre las acciones de las personas y sobre los efectos de sus acciones. Detrás de este razonamiento, está la premisa epistemológica de que la validez de una teoría depende de su poder de previsión. Pero como sabemos la fuerza de una teoría no depende de su poder de predicción. Dada la complejidad de la sociedad, la corroboración o falsación de una teoría no son relevantes porque ellas pueden ser debidas a otras mediaciones que pueden potencialmente intervenir (Lawson, 1989). Por otro lado, de la observación entre el modelo y la realidad, podría ser legítimo inferir que las personas generalmente actúan de acuerdo con principios básicos de racionalidad, pero sería un 
error tomar tal congruencia como evidencia empírica de que las personas normalmente actúan racionalmente. Y este punto es importante, pues las elaboraciones sobre vulnerabilidad que son objeto de este debate, parecería que apelan a la capacidad de proyección racional de conductas que disminuyan los impactos o las situaciones de riesgo en el interjuego Estado, Mercado y Grupos Familiares.

- Sobre el "externalismo" en la Teoría de la Escuela Racional: Algunos teóricos de la escuela racional indican su particular versión de la misma, denominándola "externalista" (Friedman, 1953; Becker, 1976), señalando con esto que han abandonado la premisa de la intencionalidad de toda acción humana. Becker (1976:112), por ejemplo, afirma que su abordaje económico: “... no considera que las unidades de decisión sean necesariamente concientes de sus esfuerzos para maximizar, o sean capaces de verbalizar o, al contrario, describan sus razones para los padrones sistemáticos en su comportamiento". Pero ese "necesariamente concientes" no borra el principio de que las personas actúan racionalmente o hacen esto $\mathrm{u}$ aquello porque adquieren habilidades o prácticas -que tienen una racionalidad- o porque estas habilidades y prácticas son producto de un cálculo conciente. Tan solo lo relativiza o entran en contradicción. Tomemos un ejemplo de Becker (1976), quien defiende que las personas viven un estilo de vida no saludable no por ignorancia sino porque otras cosas son más importantes para ellas que maximizar sus expectativas de vida. Algunas decisiones como fumar, tener una vida sedentaria, “...serían decisiones desaconsejables si el único objetivo fuese una vida larga, pero considerando que existen otras metas, ellas podrían ser informadas y en este sentido sensatas" (Becker, 1976: 114). Si el autor se considera un "externalista", nociones como objetivos, decisiones informadas, decisiones sensatas, entran en completa contradicción. Del mismo modo, si observamos las citas de los autores vernáculos, la puesta en marca de "activos", la disminución de "pasivos", las estrategias para alcanzar un lugar en la "estructura de oportunidades", obviamente hacen referencia, por elevación, al apelo de objetivos, acciones planificadas, bajo las cuales subyace un componente ineludible de racionalidad. 
- Racionalidad, Cultura y Contexto Social: En este punto cabe destacar que los teóricos ya citados no toman en cuenta enteramente el contexto cultural en el cual las personas se encuentran insertas o lo que ellas consideran ser o no una convicción fundada en la razón. El problema con la teoría de la acción racional es que ella trabaja con convicciones sobre la relación entre acción y resultado, lo que necesariamente se apoya en nociones culturales que muchas veces no son las de los sujetos a los cuales se "aplican" sus presupuestos teóricometodológicos. Respecto a las elaboraciones de Katzman et al (1999), las transformaciones en el mundo del trabajo -desempleo estructural, precarización del empleo, etcéterason señaladas pero no son relacionadas con un tipo de modelo de desarrollo, o en otras palabras, con los procesos de acumulación del capital que caracterizan los tiempos actuales. La mano oculta del mercado continúa allí, actuando, abriendo o comprimiendo la estructura de oportunidades. Los esfuerzos de familias y hogares para superar barreras -pasivos- muchas veces son infructuosos ante tales impedimentos que son de índole claramente estructural. Entonces, ¿cuál es el papel que le cabe a la comprobación empírica de acciones racionales "exitosas"? Y muchas veces ya el círculo de la exclusión hace que ni se intenten acciones racionales para poder salir del mismo. Nos encontramos frente a otra racionalidad: la guiada por la impotencia, que claro está se encuentra vinculada a dimensiones culturales y psicológicas en el marco de un modelo de desarrollo que multiplica la desigualdad económica y la inequidad política.

Retomando brevemente esta teoría, pensamos que la elaboración de Katzman (1999) y Katzman et al. (1999) reflejan en parte esta última concepción de los seres humanos como agentes racionales en toda su vida de relación y en todas sus decisiones. A modo de ejemplo: reducir sus pasivos, analizar la estructura de oportunidad e identificar los "nichos" en los cuales pueden "insertarse", fomentar sus activos de todo tipo, etc. Pero todos sabemos que no siempre actuamos racionalmente o no siempre poseemos la información necesaria para hacerlo.

En nuestra opinión existe una suerte de sintonía entre ambas propuestas, pues en definitiva, los autores citados hacen hincapié 
en la puesta en acción de los activos individuales y familiares. Es cierto que también indican procesos regresivos en el mercado y en el Estado, es decir, en componentes estructurales de la sociedad, pero no profundizan las razones por las cuales la estructura de oportunidades se amplía o adelgaza. Parecería ser que tanto mercado, Estado, sociedad, como individuos y familias poseen los mismos grados de responsabilidad, información y racionalidad sobre los escenarios de vulnerabilidad. Creemos que es en este punto relacionado con la teoría de elección racional y la cultura, donde más se aproximan ambas elaboraciones.

\section{Conclusiones}

Consideramos la propuesta de Katzman et al. (1999) algo espuria, en el entendido que subyacen a la misma elementos de la teoría de la elección racional que no son debidamente explicitados. Ya hemos realizado las críticas que esta teoría nos merece desde un punto de vista teórico pero también metodológico y epistemológico. De manera sucinta señalamos:

- Que una noción de racionalidad ajena a la cultura, en este caso de la población que se analiza, es altamente problemática. Apelamos, por el contrario, a la capacidad reflexiva colectiva que refiere a prácticas compartidas mediante las cuales las personas y familias construyen sus cursos de vida, a pesar de las limitaciones que ofrece el mercado, en palabras de los autores de referencia.

- En segundo lugar, parece no tener sentido que las acciones de personas y familias de forma general son racionales, en el sentido dado por Kaztman et al. (1999), de optimización de activos ajustada al ambiente, Estado y Mercado. La visión de que existe una y apenas una forma racional de actuar es engañosa. En general las personas no verifican continuamente la racionalidad de sus acciones.

- La capacidad reflexiva a la que apelamos es mucho más significativa, sociológicamente hablando, para asumir acciones públicas y colectivas. Existe una suerte de individualismo sociológico en el concepto de vulnerabilidad analizado, por más que se contextualice en familias de determinados segmentos sociales. 
En definitiva, la propuesta se muestra, en nuestra opinión, como un modelo de análisis que amplía el campo de la economía a lo social y cultural. Pero que en definitiva no llega a explicar las razones últimas sobre por qué la vulnerabilidad social, o por qué el binomio exclusión -vulnerabilidad no deja de ser una forma de integración social pero sumamente barbarizante. En pocas palabras, no acordamos in totum con esta propuesta por su utilidad engañosa. Utilidad engañosa pues se basa en una razón analítica -positivistaque no llega a explicar las razones últimas de tan complejos procesos sociales. Su capacidad descriptiva y su utilidad para la definición o gerenciamiento de Programas o Políticas Sociales es indudable, pero no por su utilidad debe ser evaluada su verdad, recordando en esto a Marx. Asumimos lo discutible de nuestra posición, que es colocada con el mayor de los respetos hacia los autores que han motivado estas reflexiones.

\section{Referencias bibliográficas}

Baraibar, X. (1999). Temas viejos en tiempos nuevos: aproximaciones al debate sobre exclusión social. Tesis de Maestría no publicada. Departamento de Trabajo Social. Facultad de Ciencias Sociales. UDELAR. Montevideo, Uruguay.

Beck, U. (1998). La sociedad del riesgo. Hacia una nueva modernidad. Buenos Aires: Paidós.

Becker, G. (1976). Social choice and individual values. New Haven: Yale University Press.

Castell, R. (1997). La metamorfosis de la cuestión social. Una crónica del asalariado. Buenos Aires: Paidós.

Coleman, J., (1990) Foundations of social theory. Cambridge: Harvard University Press.

De los Campos, H. (2000) El Índice de Necesidades básicas insatisfechas. Crítica de la definición oficial y propuesta de una metodología alterna. Documento de Trabajo Nro 13. Montevideo: UDELAR

Friedman, M. (1953). Essays in positive economics. Chicago: University of Chicago Press.

KAZTMAN, R. (coord.) (1999). Activos y estructuras de oportunidades. Estudios sobre las raíces de la vulnerabilidad social en Uruguay. Montevideo: CEPAL 
Lawson, T. (1990). Abstraction, tendencies an stylised facts: a realist approach to economic analysis. Cambridge Journal of Economics. 13, pp. 67- 95.

Organización Internacional del Trabajo y Fundación Ford. (1999). Vulnerabilidad, activos y exclusión en Argentina y Uruguay. Santiago, Chile: OIT

O'Malley, P. (1991). Risk and responsibility. En: A. Barry, T. Osborne y N. Rose (eds) Foucault and Political Reason. Chicago: The University of Chicago Press. P. 189-207.

Olson, J. (1965). The logic of collective action. Cambridge: Harvard University Press. 